\title{
Fully decoupled current control and energy balancing of the Modular Multilevel Matrix Converter
}

\author{
Felix Kammerer, Johannes Kolb and Michael Braun \\ Karlsruhe Institute of Technology (KIT) \\ Elektrotechnisches Institut (ETI) - Electrical Drives and Power Electronics \\ Kaiserstr. 12, 76131 Karlsruhe, Germany \\ felix.kammerer@kit.edu,www.eti.kit.edu
}

\begin{abstract}
The Modular Multilevel Matrix Converter (M3C) is a Modular Multilevel Converter topology which is suitable for high power low speed drive applications. This paper presents a fully decoupled current control which allows an independent input, output and internal balancing current control. To equalize the energy stored in the nine converter arms, an energy and balancing control is presented which includes average, horizontal, vertical and diagonal balancing control loops. Simulation results are used to verify the function of the $\mathrm{M3C}$ together with an induction motor drive system. Additionally, the proper function of the recently constructed arm PCB working as single phase multilevel STATCOM is presented. This PCB will be used for each arm in the laboratory prototype of the $\mathrm{M3C}$ in the near future.
\end{abstract}

Index Terms-Modular Multilevel Matrix Converter (M3C), current control, energy and balancing control, drive application

\section{INTRODUCTION}

The Modular Multilevel Matrix converter (M3C) shown in fig. 1 was firstly presented in [1] without the arm inductors $L$. The converter arms work as controllable voltage sources. Corresponding control principles based on the space vector modulation are described in [2] and [3]. The method is difficult to implement in a $\mathrm{M} 3 \mathrm{C}$ with a higher number of cells per arm because of the very high number of possible space vectors which increase with the number of cells per arm. Only 5 arms are allowed to be switched on simultaneously to avoid the problems of a possible parallel connection of the voltage sources. Because of this, the arm currents cannot flow continuously. For this reason in [4] the M3C is presented with arm inductors. There, the arm currents can flow continuously and the converter arms can work as controllable current sources. A feed forward control is presented and extended in [5] with a DC-circulating current for the energy balancing control. Nevertheless, the proper function is not guaranteed under all possible operating points. The first cascaded feedback control system was presented in [6]. It contains an inner arm current control and an outer arm energy control. Due to the fact that the neutral points $N_{e}$ and $N_{a}$ are not connected, the current control is overdetermined and problems will be expected during the dynamic operation. [7] shows control

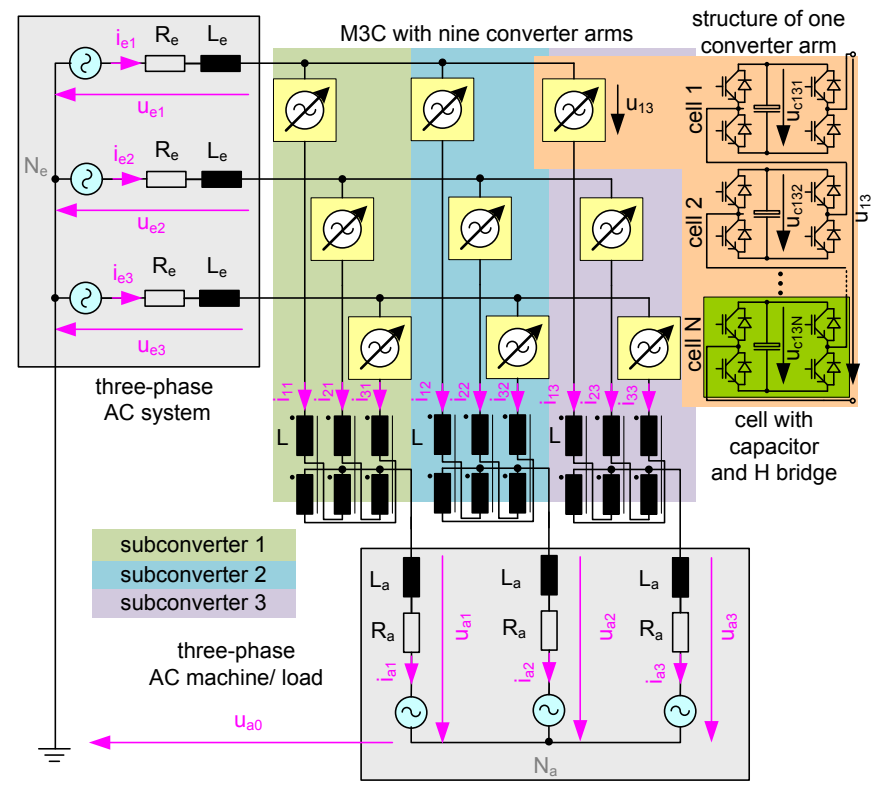

Fig. 1. Modular Multilevel Matrix Converter (M3C) with coupled three-phase z-winding arm inductors L

principles based on the power balance and dimensioning issues of the $\mathrm{M} 3 \mathrm{C}$ for high power low speed drive applications. Compared to a back-to-back configuration of two Modular Multilevel Converters for feeding a three-phase machine (see [8]-[15]) the output currents of the M3C and for this reason the possible electric torque at low nominal output frequencies is not reduced by internal balancing currents.

In [16] the $\mathrm{M} 3 \mathrm{C}$ is presented with the coupled threephase z-winding arm inductors L (fig.1) together with a cascaded vector control scheme. This control scheme allows the independent input and output current control for one subconverter. The outer energy and balancing control loops are able to guarantee the proper energy distribution in all nine converter arms with an average energy as well as horizontal and vertical balancing control loops. A method to estimate the required amount of capacitance for a given drive application is explained in [17]. Additionally, a detailed analysis of the 
coupled z-winding arm inductor, which allows considerable material savings and a better control performance, is presented.

Based on the control principles presented in [16], a fully decoupled current control is presented here with the following characteristics:

- Two controllers are used for the input current control of the M3C. This allows the independent control of the active and reactive power interchanged with the grid.

- Four controllers are used for the internal balancing currents which can be used for energy balancing and the reduction of the energy pulsation in the converter arms.

- Two controllers are used for the current control of the connected load or machine (magnetization and torque).

A diagonal balancing control is added to the outer energy and balancing control loops. Therefore, the following controllers are used for the nine converter arm energies:

- One controller is used for the average energy totally stored in the whole converter.

- Two controllers are used for the horizontal balancing control.

- Two controllers are used for the vertical balancing control.

- Additionally, two pairs of controllers are used for the two diagonal balancing controls.

This control scheme allows the proper operation of the Modular Multilevel Matrix Converter (M3C) under all possible steady state and dynamic operating points.

The M3C itself consists of 3 subconverters (fig.1). Each subconverter connects the three input phases from the grid via three converter arms and the coupled z-winding arm inductor $L$ to one output phase. The converter arms are constructed as a series connection of $\mathrm{N}$ cells which consists of an $\mathrm{H}$ bridge with four semiconductor switches, a DC-capacitor and the corresponding control electronics. The balancing of the energy inside of one converter arm is part of the modulation (see [16]). For this reason the presented control scheme is independent from the number of cells per arm N. This enables the scalability of the $\mathrm{M} 3 \mathrm{C}$ in the voltage range.

\section{Cascaded Vector Control of the M3C}

The fully decoupled control of the $\mathrm{M} 3 \mathrm{C}$ is discussed in this section. First the necessary transformation steps for the measured arm currents and arm capacitor voltages are shown. Second, the transfer functions for the different control loops are analyzed for the controller design. Finally all the cascaded control loops are explained in detail.

\section{A. Transformation of the arm currents}

[16] shows that the input and output currents of a subconverter are linearly independent because the output current is the zero sequence of the arm currents. Therefore the following transformation matrix is used to obtain the $\alpha \beta 0$-components of the subconverter arm currents:

$$
T_{i, 3 \rightarrow \alpha \beta 0}=\left[\begin{array}{ccc}
\frac{2}{3} & -\frac{1}{3} & -\frac{1}{3} \\
0 & \frac{1}{\sqrt{3}} & -\frac{1}{\sqrt{3}} \\
1 & 1 & 1
\end{array}\right]
$$

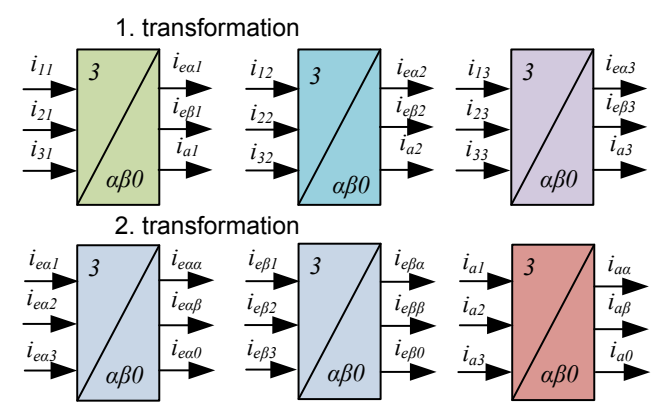

Fig. 2. Transformation of the arm currents to calculate the input, output and internal currents

This first transformation step is done for all three subconverters (fig. 2). The results are the subconverter input currents in $\alpha \beta$ components and the zero sequence component equal to the subconverter output current. In a second transformation step, using the same transformation matrix, all the $\alpha$, all the $\beta$ and all the zero sequence components are transformed again. This leads to the transformed current components shown in Table I which are used for the fully decoupled current control. The zero sequence output current $i_{a 0}$ is always zero because the neutral points $N_{e}$ and $N_{a}$ are not connected. The internal balancing currents are used for the energy balancing inside of the M3C. Additionally, it is possible to use them for a reduction of the energy pulsation inside of the converter arms (see [7]).

TABLE I

TRANSFORMED CURRENT COMPONENTS

\begin{tabular}{c|c}
\hline$i_{e \alpha 0}, i_{e \beta 0}$ & input currents \\
\hline$i_{a \alpha}, i_{a \beta}$ & output currents \\
\hline$i_{a 0}=0$ & zero sequence output current \\
\hline$i_{e \alpha \alpha}, i_{e \alpha \beta}, i_{e \beta \alpha}, i_{e \beta \beta}$ & internal balancing currents \\
\hline
\end{tabular}

\section{B. Transformation of the arm capacitor voltages}

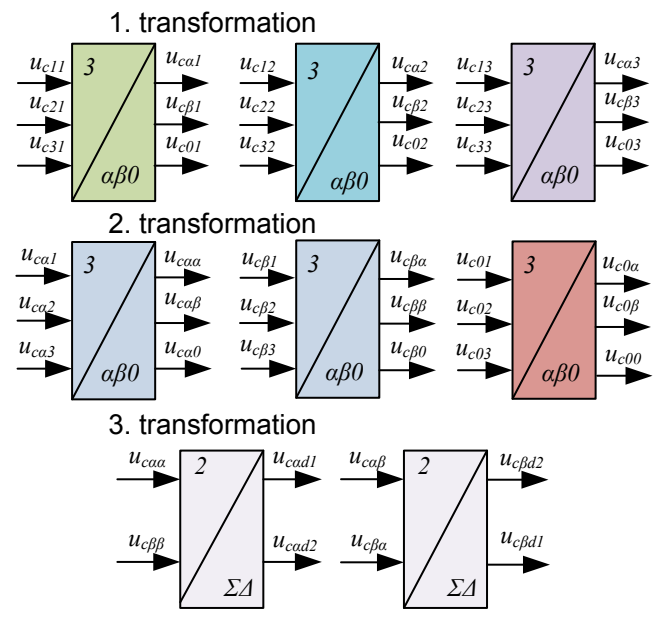

Fig. 3. Transformation of the arm capacitor voltages to calculate the average value and the horizontal, vertical and diagonal balancing components 
The measured cell capacitor voltages are summed up to calculate arm capacitor voltages:

$$
u_{c x y}=\sum_{z=1}^{N} u_{c x y z} \quad x, y \in 1,2,3
$$

After that, the arm capacitor voltages of all nine converter arms are transformed to get the average value for the energy control as well as the horizontal, vertical and diagonal components for the balancing control. Subsequently the following transformation matrix is used for all three subconverters (fig. 3) in the first step:

$$
T_{u, 3 \rightarrow \alpha \beta 0}=\left[\begin{array}{ccc}
\frac{2}{3} & -\frac{1}{3} & -\frac{1}{3} \\
0 & \frac{1}{\sqrt{3}} & -\frac{1}{\sqrt{3}} \\
\frac{1}{3} & \frac{1}{3} & \frac{1}{3}
\end{array}\right]
$$

The result is an average component and $\alpha \beta$-components for each subconverter. In a second step, all the $\alpha$, all the $\beta$ and all the zero sequence components are transformed by using the same transformation matrix. Finally, four of the nine values are transformed in a third step to get the diagonal balancing components using the following transformation matrix which calculates the sum and difference of the 2 components:

$$
T_{u, 2 \rightarrow \Sigma \Delta}=\left[\begin{array}{ll}
+\frac{1}{2} & +\frac{1}{2} \\
+\frac{1}{2} & -\frac{1}{2}
\end{array}\right]
$$

This leads to the arm capacitor voltage components described in Table II which correspond to the different balancing directions.

TABLE II

TRANSFORMED ARM CAPACITOR VOLTAGE COMPONENTS

\begin{tabular}{c|c}
\hline$u_{c 00}$ & average value for the energy control \\
\hline$u_{c 0 \alpha}, u_{c 0 \beta}$ & horizontal balancing components \\
\hline$u_{c \alpha 0}, u_{c \beta 0}$ & vertical balancing components \\
\hline$u_{c \alpha d 1}, u_{c \beta d 1}$ & diagonal balancing control 1 components \\
\hline$u_{c \alpha d 2}, u_{c \beta d 2}$ & diagonal balancing control 2 components \\
\hline
\end{tabular}

\section{Transfer functions for the current controller design}

The corresponding transfer functions for the design of the current controller are deduced in [16] for a subconverter and are given here for the $\mathrm{M} 3 \mathrm{C}$ considering the line inductances $L_{e}$ and line resistances $R_{e}$. For the internal balancing currents $i_{e \alpha \alpha}, i_{e \alpha \beta}, i_{e \beta \alpha}$ and $i_{e \beta \beta}$ the following transfer function is used:

$$
G_{i_{\text {exy }}}(s)=\frac{i_{\text {exy }}(s)}{u_{\text {iexy }}(s)}=\frac{1}{s L} \quad x, y \in\{\alpha, \beta\}
$$

$L$ is the effective arm inductance for the subconverter input currents. Due to the parallel connection of the subconverters the transfer function for the input currents $i_{e \alpha 0}$ and $i_{e \beta 0}$ is:

$$
G_{i_{e x 0}}(s)=\frac{i_{e x 0}(s)}{u_{i e x 0}(s)}=\frac{1}{R_{e}} \cdot \frac{1}{1+s \frac{L_{e}+\frac{L}{3}}{R_{e}}} \quad x \in\{\alpha, \beta\}
$$

For the output currents $i_{a \alpha}$ and $i_{a \beta}$ only the transfer function of the connected machine is relevant:

$$
G_{i_{a x}}(s)=\frac{i_{a x}(s)}{u_{a x}(s)}=\frac{1}{R_{a}} \cdot \frac{1}{1+s \frac{L_{a}}{R_{a}}} \quad x \in\{\alpha, \beta\}
$$

This is possible because of the coupled three-phase $\mathrm{z}$-winding arm inductors $L$ which aren't magnetically effective to the output currents [16], [17]. In case of the use of single arm inductors $L$ (see [4]) they have to be considered with $\frac{L}{3}$ in the transfer function for the output currents:

$$
G_{i_{a x}}^{*}(s)=\frac{i_{a x}(s)}{u_{a x}(s)}=\frac{1}{R_{a}} \cdot \frac{1}{1+s \frac{L_{a}+\frac{L}{3}}{R_{a}}} \quad x \in\{\alpha, \beta\}
$$

\section{Transfer functions for the energy and balancing controller design}

The transfer function which is used for the controller design of the energy control loop is defined as:

$G_{u_{c 00}}(s)=\frac{u_{c 00}(s)}{i_{e d 0}(s)}=\frac{\hat{U}_{e}}{6 \cdot u_{c 00}} \cdot \frac{1}{s C_{a r m}} \approx \frac{\hat{U}_{e}}{6 \cdot u_{c 00}^{*}} \cdot \frac{N}{s C_{c e l l}}$

The gain of this transfer function depends on the actual voltage $u_{c 00}$ and the input voltage amplitude $\hat{U}_{e}$. For this reason the gain of the controller needs to be adapted for different operating points to allow a linear controller design. Considering that the voltage variation (which is equal to a gain variation) is small compared to the average reference value $u_{c 00}^{*}$, this value is used for the calculations instead of using the actual arm capacitor voltage $u_{c 00}$. Additionally it is assumed that the energy is distributed equally to all cells inside of one converter arm by the modulator [16].

Using this considerations the transfer functions for the horizontal and diagonal balancing control are calculated to:

$$
\begin{gathered}
G_{u_{c 0 x}}(s)=\frac{u_{c 0 x}(s)}{i_{e x y}(s)} \approx \frac{\hat{U}_{e}}{2 \cdot u_{c 00}^{*}} \cdot \frac{N}{s C_{c e l l}} \quad x, y \in\{\alpha, \beta\} \\
G_{u_{c x d 1 / 2}}(s)=\frac{u_{c x d 1 / 2}(s)}{i_{e x y}(s)} \approx \frac{\hat{U}_{e}}{2 \cdot u_{c 00}^{*}} \cdot \frac{N}{s C_{c e l l}} \quad x, y \in\{\alpha, \beta\}
\end{gathered}
$$

For the vertical balancing control different transfer functions are used depending on the balancing method (see section II-G). For balancing with the zero sequence output voltage $u_{a 0}$ the following transfer function is used:

$$
G_{u_{c x 0}}(s)=\frac{u_{c x 0}(s)}{u_{a 0}(s)} \approx \frac{\hat{i}_{e}}{6 \cdot u_{c 00}^{*}} \cdot \frac{N}{s C_{c e l l}} \quad x \in\{\alpha, \beta\}
$$

In case of balancing with the internal currents the transfer function is defined as:

$$
G_{u_{c x 0}}(s)=\frac{u_{c x 0}(s)}{i_{e x y}(s)} \approx \frac{\hat{U}_{a}}{2 \cdot u_{c 00}^{*}} \cdot \frac{N}{s C_{c e l l}} \quad x, y \in\{\alpha, \beta\}
$$

Therefore the gain for the vertical balancing controllers needs to be adapted depending on the balancing method. 


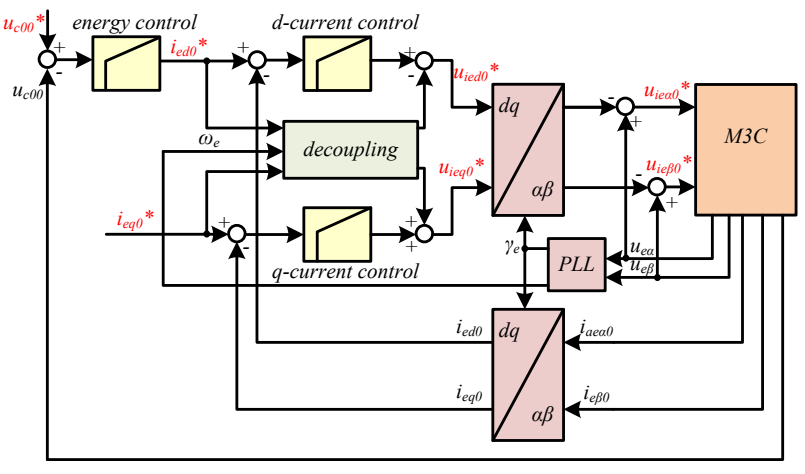

Fig. 4. Energy and input current control

\section{E. Energy and input current control}

The input current control can be done in a stationary dqreference frame using the phase angle $\gamma_{e}$ of the grid voltage $u_{e}$ (fig. 4). This allows the independent control of the active and reactive power generating currents $i_{e d 0}$ and $i_{e q 0}$ with a pair of PI-controllers (see [18], [19]). A feed forward decoupling is used to eliminate the cross-coupling. The reference value for the active power generating current $i_{e d 0}^{*}$ is given by the energy controller. This PI-controller maintains the energy stored in the nine converter arms at a constant level. The reference value for the reactive power generating current $i_{e q 0}^{*}$ can be set to zero or a desired value for working additionally as a STATCOM to support the grid with reactive power (see [20]).

\section{F. Cascaded Motor control}

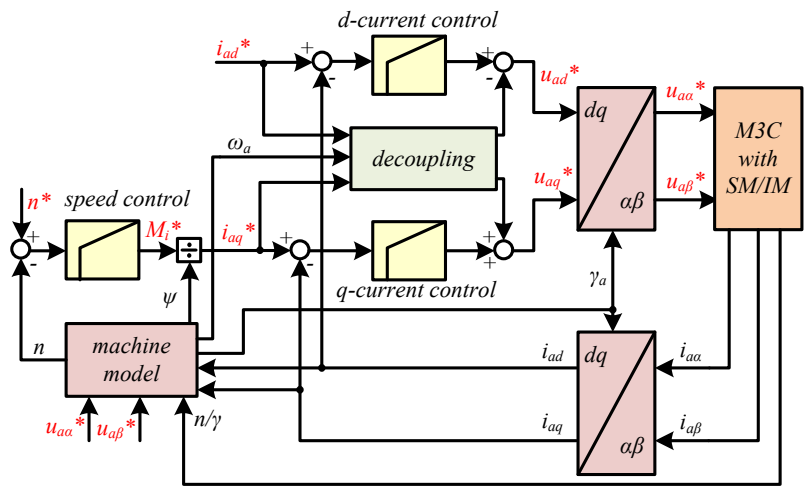

Fig. 5. Field-/rotor-oriented control of a three phase machine connected to the $\mathrm{M} 3 \mathrm{C}$

The control of the electrical machine connected to the M3C can be realized with a conventional field- or rotor-oriented control (fig. 5) depending on the machine type. Therefore the measured and transformed output currents $i_{a \alpha}$ and $i_{a \beta}$ are rotated into the corresponding stationary reference frame to control the magnetic flux and electric torque generating currents independently with a pair of PI-controllers. A feed forward decoupling is used to eliminate the cross-coupling. The torque current reference value $i_{a q}^{*}$ is given by an superimposed speed controller which has to be designed depending on the requirements of the mechanical process.
In case of using the M3C to connect two three-phase grids with different frequencies $f_{e}$ and $f_{a}$ the speed controller can be replaced by active and reactive power controllers to give the reference values for the currents $i_{a d}^{*}$ and $i_{a q}^{*}$. To feed an island grid it is also possible to generate directly the output voltage reference values $u_{a d}^{*}$ and $u_{a q}^{*}$ without any current controller.

\section{G. Balancing and internal current control loops}

Four pairs of PI-controllers are used to balance the energy stored in the nine converter arms (fig. 6). Each pair controls one of the four balancing directions in $\alpha \beta$-coordinates. The measured and transformed voltage components are filtered to control only the average values due to the intrinsic energy pulsation in the converter arms [17]. Here, additional benefits of the proposed transformation can be obviously seen: During symmetrical operation of the M3C each of the four different angular frequency components $\omega_{e}, \omega_{a}$ and $\omega_{e} \pm \omega_{a}$ is related to one of the balancing directions which allows an easier filter design. Only during the balancing process a small crosscoupling occurs and needs to be investigated to design a feedforward decoupling in the future.

The horizontal balancing control distributes the energy between the three subconverters. Therefore, the values $u_{c 0 \alpha}$ and $u_{c 0 \beta}$ are filtered and compared with the reference values which are set to zero to eliminate any horizontal unbalance. To reduce the energy pulsation with $2 \omega_{a}$ at very low output frequencies also non-zero reference values are possible [7]. The current reference values from the horizontal balancing control are rotated into the positive sequence reference frame using the grid angle $\gamma_{e}$ to generate active power components together with the grid voltage $u_{e}$ which distributes the energy horizontally.

To eliminate the diagonal unbalance the values $u_{c \alpha d 1}$, $u_{c \beta d 1}, u_{c \alpha d 2} u_{c \beta d 2}$ are filtered and controlled to zero. The resulting reference current amplitudes are transformed using the following transformation matrix:

$$
T_{u, \Sigma \Delta \rightarrow 2}=\left[\begin{array}{ll}
+1 & +1 \\
+1 & -1
\end{array}\right]
$$

After that, they are rotated into the negative sequence reference frame using the grid angle $\gamma_{e}$ to generate active power components together with the input voltage $u_{e}$ which distributes the energy diagonally.

The values $u_{c \alpha 0}, u_{c \beta 0}$ are filtered and controlled to zero for the vertical balancing control. Here, different strategies are possible: One of them is the use of negative sequence input currents which generates active power components together with the input voltage (see [7]). This is not considered here to prevent the grid from unsymmetrical line currents. In [16] a vertical balancing control using a zero sequence voltage $u_{a 0}$ with line frequency $\omega_{e}$ is presented. This control generates active power components together with the input currents $i_{e \alpha / \beta 0}$. For this reason a small reactive power generating current has to be used in case of no-load operation where the active power generating currents are very small. 


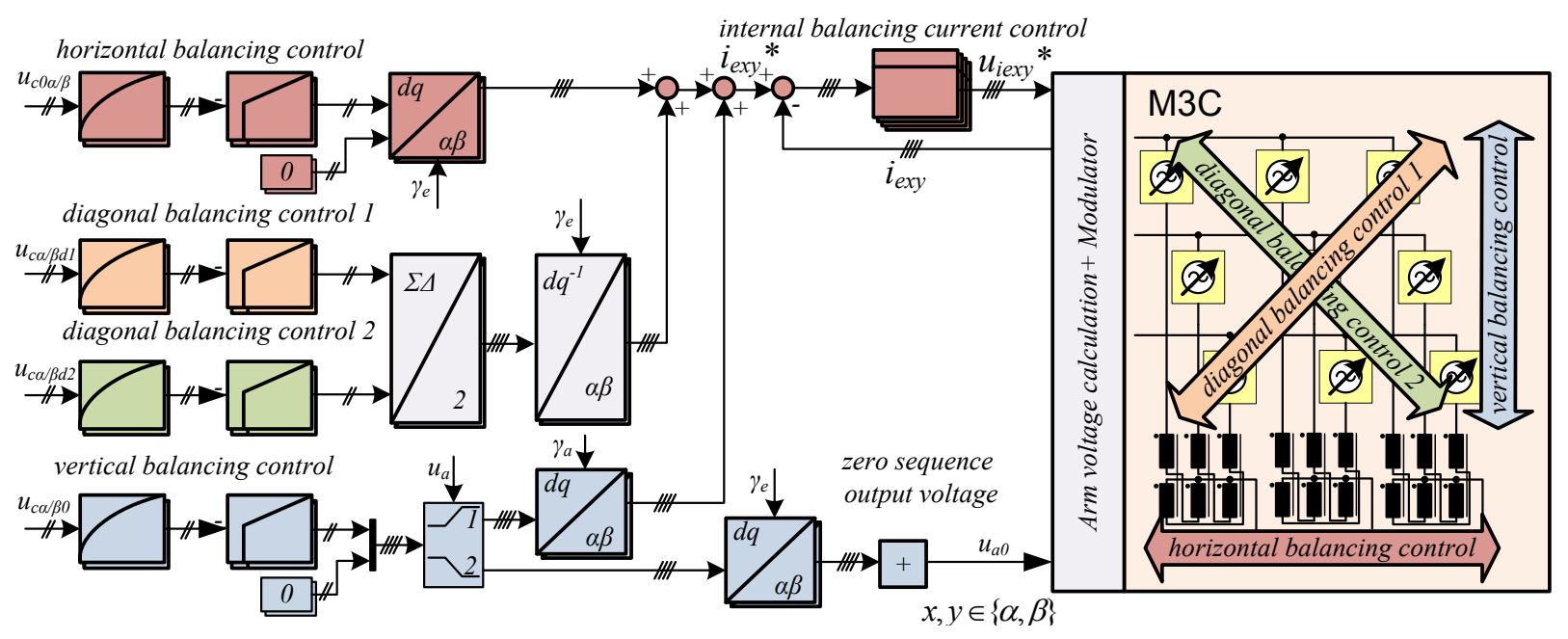

Fig. 6. Outer balancing control loops with inner internal balancing current controllers

A third possibility is presented here which generates active power components together with the output voltages $u_{a \alpha \beta}$. Therefore the reference current amplitudes are rotated into a positive sequence reference frame using the angle $\gamma_{a}$ from the output voltages $u_{a \alpha \beta}$. This method is very attractive for higher output voltages $u_{a} \alpha \beta$ to avoid a zero sequence voltage $u_{a 0}$ which reduces the maximum output voltage. In drive applications the two methods can be switched over: At low output voltages the zero sequence voltage $u_{a 0}$ and at higher output voltages the internal balancing currents can be used. In case of grid applications, the internal balancing currents can be used for the vertical balancing to avoid any balancing currents or zero sequence voltage at the converter terminals.

The resulting internal balancing reference currents from the four different balancing control loops are summarized to get the reference values $i_{e \alpha \alpha}^{*}, i_{e \alpha \beta}^{*}, i_{e \beta \alpha}^{*}, i_{e \beta \beta}^{*}$ for the four internal balancing current control loops. The four currents are controlled using only Proportional (P)-controllers in the stationary reference frame due to the different frequency components which do not allow using a rotating reference frame. The resulting control deviations are small and eliminated by the superposed balancing control. Additionally, they do not influence neither the input nor the output currents.

\section{ARM REFERENCE VOLTAGE CALCULATION}

The voltages generated by the input current control, by the output current control and by the internal balancing control are transformed together with the zero sequence output voltage $u_{a 0}$ to calculate the arm reference voltages. They have to be generated by the nine converter arms of the $\mathrm{M} 3 \mathrm{C}$. The following transformation matrix is used two times (fig. 7):

$$
T_{u, \alpha \beta 0 \rightarrow 3}=\left[\begin{array}{ccc}
1 & 0 & 1 \\
-\frac{1}{2} & \frac{\sqrt{3}}{2} & 1 \\
-\frac{1}{2} & -\frac{\sqrt{3}}{2} & 1
\end{array}\right]
$$

The $\alpha \beta 0$-components of the three subconverters are calculated in a first step. All the $\alpha$, all the $\beta$ and all the zero-sequence

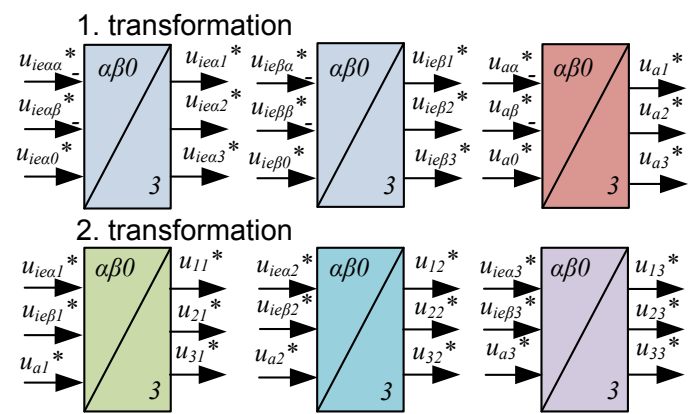

Fig. 7. Arm reference voltage calculation using 2 transformation steps

components are transformed together in the second step to get the nine arm reference voltages. These voltages are sent to the modulator which selects the corresponding switching regarding the balancing of the energy of the cells inside of one converter arm [16].

\section{Simulation Results}

TABLE III

SIMULATION PARAMETERS OF THE M3C WITH INDUCTION MACHINE (IM)

\begin{tabular}{c|c}
\hline Parameter & Value \\
\hline sampling rate & $f_{t}=8 \mathrm{kHz}$ \\
\hline input voltage amplitude & $\hat{U}_{e}=325 \mathrm{~V}$ \\
\hline input frequency & $f_{e}=50 \mathrm{~Hz}$ \\
\hline arm capacitance & $C=880 \mu \mathrm{F}$ \\
\hline reference arm capacitor voltage & $u_{c 00}^{*}=650 \mathrm{~V}$ \\
\hline input inductance & $L_{e}=2 \mathrm{mH}$ \\
\hline arm inductance & $L=750 \mu \mathrm{H}$ \\
\hline IM nominal current & $I_{N}=22.5 \mathrm{~A}$ \\
\hline IM nominal power & $P_{N}=11 \mathrm{~kW}$ \\
\hline IM nominal speed & $n_{N}=1440 \frac{1}{\mathrm{~min}}$ \\
\hline IM nominal torque & $M_{N}=73 \mathrm{Nm}$ \\
\hline IM pole pairs & 2
\end{tabular}



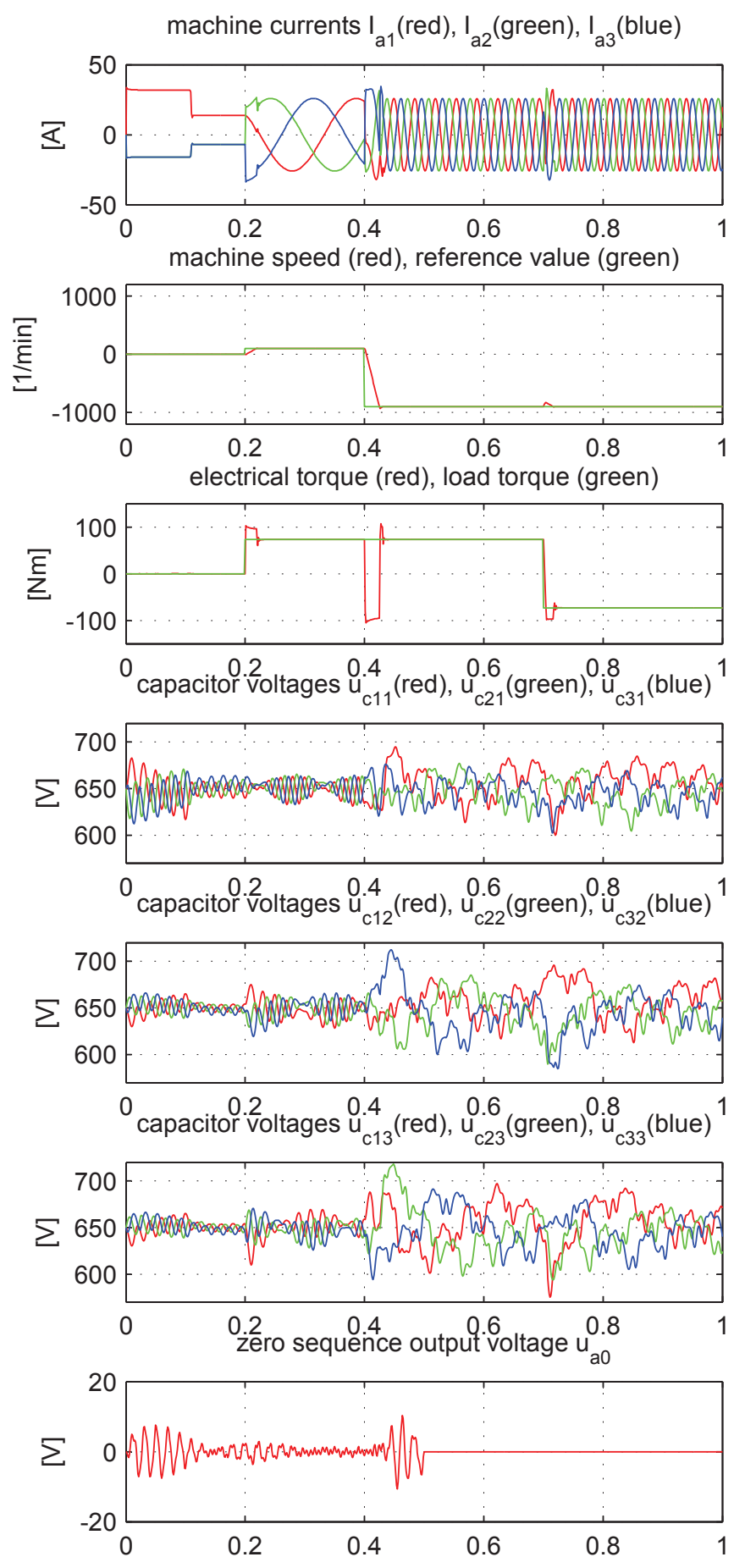

input currents $\mathrm{I}_{\mathrm{e} 1}(\mathrm{red}), \mathrm{I}_{\mathrm{e} 2}$ (green), $\mathrm{I}_{\mathrm{e} 3}$ (blue)
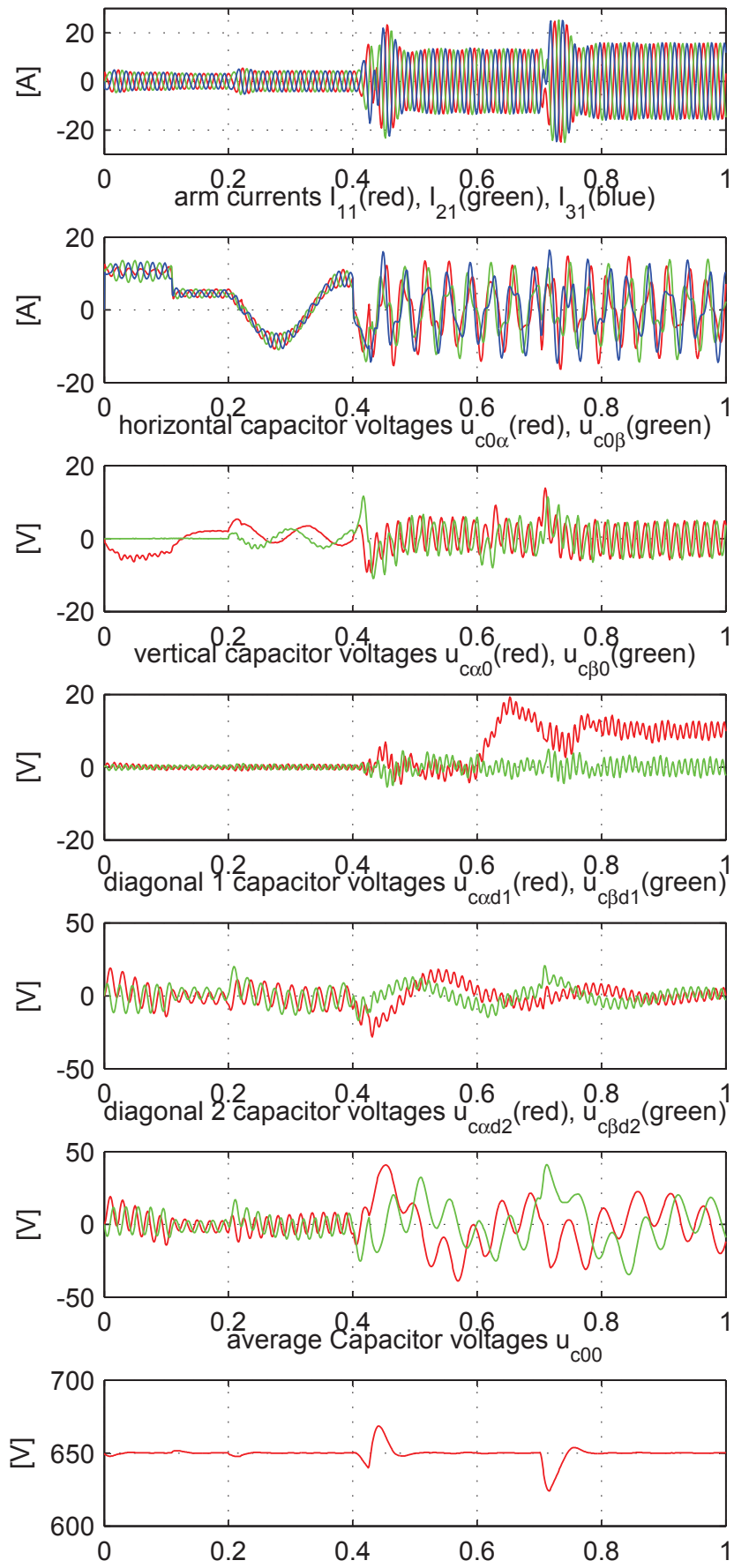

Fig. 8. Simulation results: Modular Multilevel Matrix Converter with Induction Machine

A time discrete average model of the $\mathrm{M} 3 \mathrm{C}$ has been implemented with MATLAB/Simulink to feed an induction machine (IM). This allows to test the theoretical results and the calculations for the design of the low voltage laboratory prototype which is under construction. The used parameters are given in table III. Fig. 8 shows the results: At $t=0 \mathrm{~s}$ a magnetization current $I_{a d}=\sqrt{2} \cdot I_{N}=32 \mathrm{~A}$ is switched on to generate the nominal flux $\Psi_{N}=1.15 \mathrm{Vs}$. Once the nominal flux is reached, the magnetization current is set to its nominal value $I_{a d}=I_{a d N}=13 \mathrm{~A}$. At $t=0.2 \mathrm{~s}$ a load step with $M_{l}=73 \mathrm{Nm}$ occurs and the speed reference value is set to $n^{*}=200 \frac{1}{\mathrm{~min}}$. The machine accelerates with the maximum electric torque which was set to $M_{i}=1.5 \cdot M_{N}$. At $t=0.4 \mathrm{~s}$ the speed reference value is set to $n^{*}=-1000 \frac{1}{m i n}$, therefore the direction of the power flow changes. At $t=0.5 \mathrm{~s}$ the vertical balancing mode is changed from using the zero 
sequence voltage $u_{a 0}$ to the internal balancing currents. This is advantageous for higher output voltages which occurs at higher machine speeds due to the higher inner machine voltages. At $t=0.7 \mathrm{~s}$ the load torque changes to $M_{l}=-73 \mathrm{Nm}$ and the machine again works as a motor. The arm currents contain input and output current components and the internal balancing currents which are quite low.

It can be seen that the control system is able to maintain the average arm capacitor voltage at their reference value $u_{c 00}^{*}=650 \mathrm{~V}$. Due to the intrinsic energy pulsation corresponding to the reactive power components (see [17]) the balancing components of the four directions are oscillating around their average values which normally are controlled to zero. At $t=0.6 \mathrm{~s}$ the reference value for the vertical unbalance in $\alpha$-direction is changed from $U_{c \alpha 0} *=0 \mathrm{~V}$ to $U_{c \alpha 0} *=10 \mathrm{~V}$ for demonstrating the function of the balancing control. The vertical balancing control therefore changes the energy distribution inside of $\mathrm{M} 3 \mathrm{C}$ in a manner that the arm capacitor voltages $u_{c 11}, u_{c 12}$ and $u_{c 13}$ are $10 \mathrm{~V}$ higher and the other arm capacitor voltages $5 \mathrm{~V}$ lower than the average value. This exemplary result is also valid for the other balancing directions and for this reason an arbitrary energy distribution inside of the $\mathrm{M} 3 \mathrm{C}$ can be set with the presented control Nevertheless this is normally not used to get a symmetrical energy distribution inside of the $\mathrm{M} 3 \mathrm{C}$.

\section{CONSTRUCTION OF A LABORATORY PROTOTYPE}

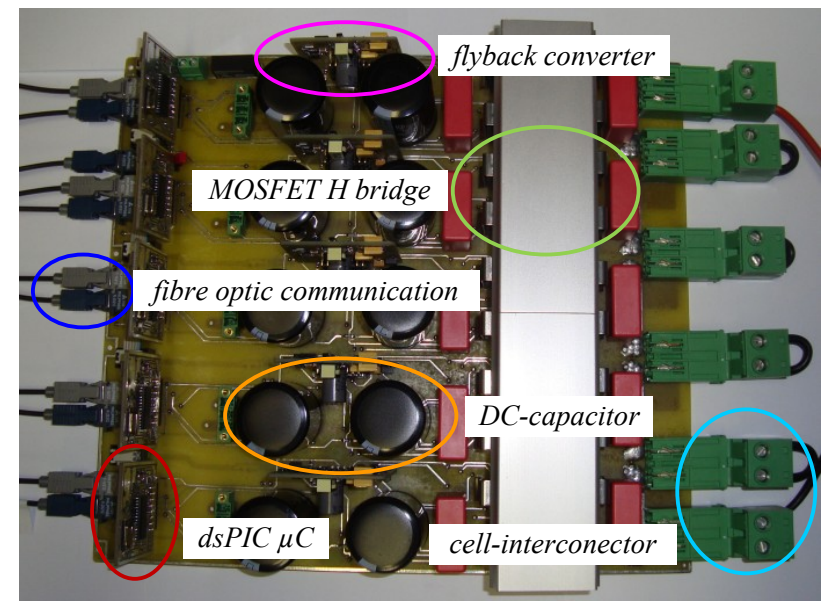

Fig. 9. Converter arm PCB with 5 cells for the laboratory prototype

A low voltage M3C laboratory prototype is under construction to verify the theoretical and simulation results. Fig 9 shows one PCB for one of nine converter arms. It consists of $\mathrm{N}=5$ cells with a MOSFET $\mathrm{H}$ bridge and an electrolytic capacitor with a voltage rating of $u_{c, \max }=160 \mathrm{~V}$. The gate drivers of one cell are supplied by a flyback converter, which is powered from the local capacitor. Therefore no external galvanic isolated power supply is necessary for the cell electronics. A Microchip dsPIC30F3010 microcontroller [21] is used for generating the gate signals and for the cell capacitor voltage sensing. The signals are transmitted via fibre optics between the modulator FPGA and the microcontroller and via RS232 UARTs. The FPGA itself selects the switching cells and sends the duty cycles to all microcontrollers. Additionally the capacitor voltages from the cells are summarized to get the nine arm capacitor voltages. The presented control itself will be executed in a digital signal processor.

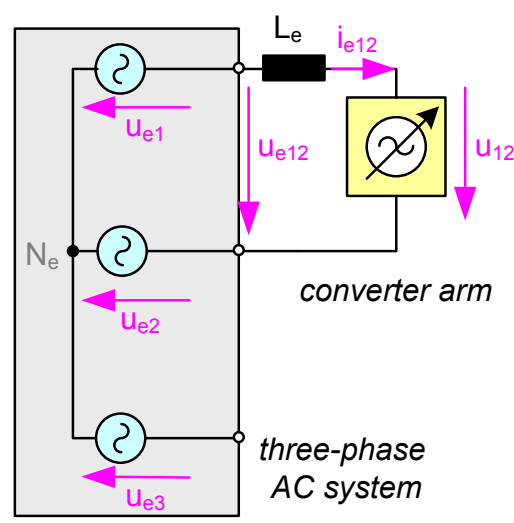

Fig. 10. Circuit for arm PCB testing as single phase STATCOM

To test a single converter arm PCB before constructing the other eight, the test circuit shown in fig. 10 is used. The converter arm PCB works as single phase STATCOM injecting capacitive or reactive currents into the $400 \mathrm{~V}, 50 \mathrm{~Hz}$ public grid. The active current is very low because he has to compensate only the losses inside of the converter arm which are very low. Fig. 11 shows the experimental results: The arm PCB injects a capacitive current with the Amplitude $\hat{I}_{e 12}=12 A$ into the grid. Due to the multilevel waveform with 11-levels generated by the 5 cells, the current has only a very small ripple caused by the switching of the cells.

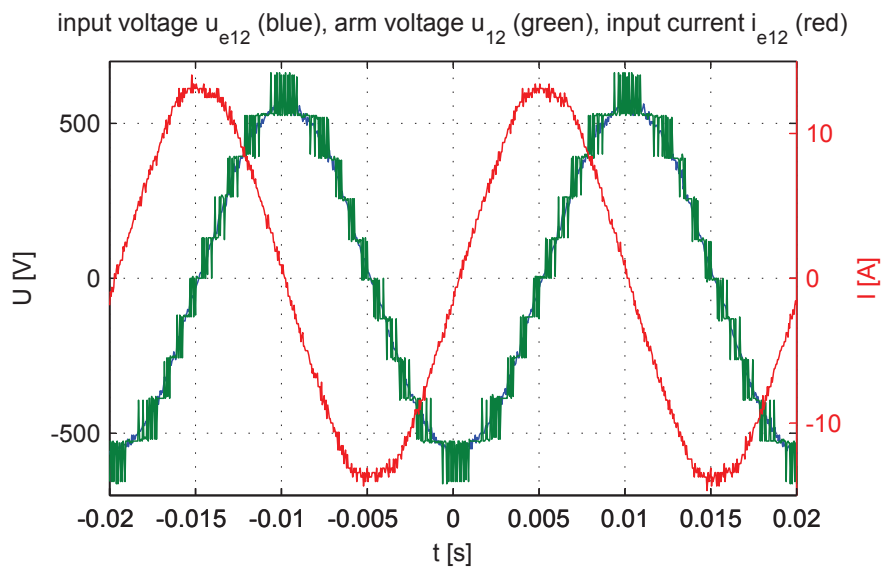

Fig. 11. Experimental results: arm PCB working as single phase STATCOM

\section{CONCLUSION}

In this paper, a fully decoupled current control and energy balancing for the Modular Multilevel Matrix Converter (M3C) is presented. It allows an equal energy distribution inside of the $\mathrm{M} 3 \mathrm{C}$ using horizontal, vertical and diagonal balancing 
control loops. The average value is controlled by an energy controller. The transformed current control allows to control independently the input currents, output currents and internal balancing currents. Additionally, a novel vertical balancing method is presented which allows the operation of the M3C without any zero sequence voltage. Simulation results are given to show the proper function of the system together with an induction machine even under dynamic changes of the operation point. Finally, the successful test of the arm PCB as single phase STATCOM which is used for the construction of the low voltage laboratory prototype is shown.

\section{ACKNOWLEDGMENT}

The authors would like to thank the DFG (German Research Foundation) which supports this research project.

\section{REFERENCES}

[1] R. Erickson and O. Al-Naseem, "A new family of matrix converters," in Industrial Electronics Society, 2001. IECON '01. The 27th Annual Conference of the IEEE, vol. 2, 2001, pp. 1515 -1520 vol.2.

[2] S. Angkititrakul and R. Erickson, "Control and implementation of a new modular matrix converter," in Applied Power Electronics Conference and Exposition, 2004. APEC '04. Nineteenth Annual IEEE, vol. 2, 2004, pp. 813 - 819 vol.2.

[3] _ - "Capacitor voltage balancing control for a modular matrix converter," in Applied Power Electronics Conference and Exposition, 2006. APEC '06. Twenty-First Annual IEEE, march 2006, p. 7 pp.

[4] C. Oates, "A methodology for developing chainlink converters," in Power Electronics and Applications, 2009. EPE '09. 13th European Conference on, sept. 2009, pp. $1-10$.

[5] C. Oates and G. Mondal, "Dc circulating current for capacitor voltage balancing in modular multilevel matrix converter," EPE 2011 Birmingham, aug. 2011.

[6] D. C. Ludois, J. K. Reed, and G. Venkataramanan, "Hierarchical control of bridge-of-bridge multilevel power converters," Industrial Electronics, IEEE Transactions on, vol. 57, no. 8, pp. 2679 -2690, aug. 2010.

[7] A. Korn, M. Winkelnkemper, P. Steimer, and J. Kolar, "Direct modular multi-level converter for gearless low-speed drives," EPE 2011 Birmingham, aug. 2011.
[8] M. Hiller, D. Krug, R. Sommer, and S. Rohner, A new highly modular medium voltage converter topology for industrial drive applications. Power Electronics and Applications, 2009. EPE 09. 13th European Conference on, sept. 2009, pp. 1 10, 2009.

[9] A. Korn, M. Winkelnkemper, and P. Steimer, Low Output Frequency Operation of the Modular Multi-Level Converter. IEEE Energy Conversion Congress and Exposition 2010, Atlanta, 2010.

[10] A. Antonopoulos, K. Ilves, L. Ängquist, and H.-P. Nee, On Interaction between Internal Converter Dynamics and Current Control of HighPerformance High-Power AC Motor Drives with Modular Multilevel Converters. IEEE Energy Conversion Congress and Exposition 2010, Atlanta, 2010.

[11] M. Hagiwara, H. Akagi, and K. Nishimura, A Medium-Voltage Motor Drive With a Modular Multilevel PWM Inverter. Power Electronics, IEEE Transactions on, vol. PP, no. 99, pp. 1 1,, 2010.

[12] J. Kolb, F. Kammerer, and M. Braun, A novel control scheme for low frequency operation of the Modular Multilevel Converter. PCIM Europe, Nuremberg, Germany, 2011.

[13] - Straight forward vector control of the Modular Multilevel Converter for feeding three-phase machines over their complete frequency range. IECON 2011 - 37th Annual Conference on IEEE Industrial Electronics Society, Melbourne, 2011.

[14] - Modulare Multilevelumrichter für Antriebssysteme - Chancen und Herausforderungen/Modular Multilevel Converters for drive systems prospects and challenges. SPS/IPC/DRIVES 2011, Kongress, Elektrische Automatisierung, Nürnberg, 2011.

[15] _ Operating performance of Modular Multilevel Converters in drive applications. PCIM Europe, Nuremberg, Germany, 2012.

[16] F. Kammerer, J. Kolb, and M. Braun, "A novel cascaded vector control scheme for the modular multilevel matrix converter," IECON 2011 Melbourne, Nov. 2011.

[17] — Optimization of the passive components of the Modular Multilevel Matrix Converter for Drive Applications. PCIM Europe, Nuremberg, Germany, 2012.

[18] F. Becker, A. Scherer, J. Weigold, and M. Braun, "A simple indirect voltage sensing method for line-connected inverters," in Power Electronics and Applications, 2007 European Conference on, sept. 2007, pp. 1 -7.

[19] I. Papic, P. Zunko, D. Povh, and M. Weinhold, "Basic control of unified power flow controller," Power Systems, IEEE Transactions on, vol. 12, no. 4, pp. $1734-1739$, nov 1997.

[20] N. Hatano and T. Ise, "Control scheme of cascaded h-bridge statcom using zero-sequence voltage and negative-sequence current," Power Delivery, IEEE Transactions on, vol. 25, no. 2, pp. $543-550$, april 2010.

[21] Microchip, dsPIC30F3010/3011 Data Sheet, 2008. 